\title{
Comparison of the Shear Bond Strength of Metal Orthodontic Brackets Bonded to Long-term Water-aged and Fresh Porcelain and Composite Surfaces
}

\author{
Yeşim Kaya' (D), Beyza Ünalan Değirmenci (D), Alperen Değirmenci ${ }^{3}$ (D) \\ 'Department of Orthodontics, Yüzüncü Yıl University School of Dentistry, Van, Turkey
²Department of Prosthodontics, Yüzüncü Yıl University School of Dentistry, Van, Turkey
${ }^{3}$ Department of Restorative Dentistry, Yüzüncü Yıl University School of Dentistry, Van, Turkey
}

Cite this article as: Kaya Y, Ünalan Değirmenci B, Değirmenci A. Comparison of the Shear Bond Strength of Metal Orthodontic Brackets Bonded to Longterm Water-aged and Fresh Porcelain and Composite Surfaces. Turk J Orthod 2019; 32(1): 28-33.

\begin{abstract}
Objective: The aim of the present study was to compare the shear bond strength (SBS) of metal orthodontic brackets bonded to longterm water-aged and fresh porcelain and composite surfaces.

Methods: One porcelain (Vitadur Alpha (VA)) and three composite (Filtek Ultimate (FU), Tetric EvoCeram (TEC), and Gradia Direct Anterior (GDA)) materials were evaluated in the present study. First, 10 discs from each material were prepared and subjected to the aging procedure for 5 years. Then, for comparison, another 10 discs from each material were prepared as fresh surfaces and stored in distilled water for $24 \mathrm{~h}$. Metal brackets were bonded to the prepared disc surfaces, and after being stored in water for $24 \mathrm{~h}$, they were subjected to shear bond test using a universal testing machine. Adhesive remnant index (ARI) scores were obtained by examining the disc surfaces under a stereomicroscope at 10x magnification. Kruskal-Wallis test was used to compare the aged and fresh groups.

Results: Although the difference between the SBS between the aged and fresh groups with VA, FU, and TEC was not significant, the SBS was significantly higher in the fresh group with GDA. With regard to ARI scores, there was no significant difference between the aged and fresh groups with FU and GDA, whereas the ARI scores of the aged groups with VA and TEC were higher.
\end{abstract}

Conclusion: It was concluded that the aged restoration materials have a distinctive influence on the SBS of metal orthodontic brackets.

Keywords: Aging, porcelain, composite, bond strength, metal brackets

\section{INTRODUCTION}

The increase in the number of adult patients receiving orthodontic treatment presents new problems to the orthodontist (1). Inadequacy of the bond strength between the orthodontic attachments and teeth or restorations to withstand orthodontic forces will lead to low success rate with adverse consequences on the cost and duration of orthodontic treatment, efficiency of appliance, and patient comfort $(2,3)$. The presence of amalgam, composite, and porcelain restorations in the adult dentition causes difficulties in bonding of orthodontic attachments and in obtaining adequate bond strength $(3,4)$. Among these, porcelain and composite restorations are preferred more than amalgam due to their aesthetic appearance $(4,5)$.

While the porcelain materials, which have an inert structure, do not change very much in the oral environment, composite materials suffer degradation due to mechanical and chemical interactions $(1,5)$. The chemical bonding of a composite resin to another composite resin surface is enabled by the unreactive methacrylate groups, which are found in the oxygen-inhibited layer of the non-polymerized resin $(4,6)$. However, the reduction of these unreactive methacrylate groups with time and the intervention of instruments for polishing of composites

Address for Correspondence: Yeşim Kaya, Department of Orthodontics, Yüzüncü Yıl University School of Dentistry, Received: April 27, 2018 Van, Turkey 
reduce the bond strength between aged and fresh composite resins $(6,7)$. For this reason, the aging of restorations is very important with regard to orthodontic bonding.

The aging process is aimed to reproduce the hydrolytic degradation that occurs in the resin matrix and the silane-coated inorganic fillers formed under oral environment conditions (7). Although there is no standardized simulation method for aging, storage in liquid media, such as citric acid, ethanol, and water, immersion in boiling water, thermocycling, accelerated aging, and artificial saliva have been used $(7,8)$. In addition, studies evaluating the bond strength of aged restorations have reported that the duration of aging and the material itself are more important than the storage medium (9-11).

The aim of the present study was to compare the shear bond strength (SBS) of metal orthodontic brackets bonded to longterm water-aged and fresh porcelain and composite surfaces. To the best of our knowledge, there are no studies in the literature that have compared the SBS of metal orthodontic brackets bonded to aged and fresh porcelain and composite surfaces. The null hypothesis is that the aging processes of porcelain and composite surfaces have no effect on SBS of metal orthodontic brackets.

\section{METHODS}

Vitadur Alpha (VA) porcelain and nanofilled Filtek Ultimate (FU), nanohybrid Tetric EvoCeram (TEC), and microhybrid Gradia Direct Anterior (GDA) composites were evaluated in the present study. The details of the porcelain and composite materials used in the study are shown in Table 1. A stainless-steel metal mold with $2 \mathrm{~mm}$ long internal concavity with $1 \mathrm{~cm}$ diameter was used to prepare the porcelain and composite discs.

Porcelain disc preparation: VA porcelain discs were prepared by mixing $0.5 \mathrm{~g}$ of ceramic powder and $0.18 \mathrm{~g}$ of model liquid until a homogeneous slurry was obtained in accordance with the manufacturer's instructions. The obtained slurry was poured into the stainless-steel mold on a vibrator to avoid any residual air inside. Then, in the dental porcelain furnace (Centurion Q200; Ney, Yucaipa, CA, USA), the following baking cycle was performed: the slurry was dried at $600^{\circ} \mathrm{C}$ for $1 \mathrm{~min}$, heated to $960^{\circ} \mathrm{C}$ with full vacuum at $60^{\circ} \mathrm{C} / \mathrm{min}$, kept at $960^{\circ} \mathrm{C}$ for $1 \mathrm{~min}$, and then removed from the furnace and allowed to cool down at room temperature.

Composite disc preparation: The composite discs were prepared by putting the resin into the metal mold by placing the polyester matrix bands on the top and at the bottom and compressing between these two lamellae. Then, using an Elipar FreeLight S10 device (3M ESPE Dental Products, St. Paul, MN, USA), the resin was polymerized in a single direction for $20 \mathrm{~s}$. After every five polymerization processes, the intensity of the light source was controlled by the intensity meter on the device. All porcelain and composite discs that were removed from the metal mold were subjected to surface treatment using 600, 800,1000 , and 1200 grit abrasive paper (Atlas, Kocaeli, Turkey), respectively.

At first, 10 discs were prepared from each material $(n=40)$ and subjected to the aging procedure in distilled water for 5 years. Then, before initiating the study, 10 new discs were prepared from each material and stored in distilled water for $24 \mathrm{~h}$ for comparison.

\section{Bracket Bonding Procedures}

Porcelain disc surface preparation: The porcelain disc surfaces were conditioned with 9.6\% hydrofluoric acid (Ultradent Products, South Jordan, UT, USA) for $60 \mathrm{~s}$, then washed with water for $60 \mathrm{~s}$, and dried with oil-free air spray (5).

Composite disc surface preparation: The composite disc surfaces were conditioned with $37 \%$ phosphoric acid (Condicionador Dental Gel; Dentsply, Rio de Janeiro, Brazil) for $30 \mathrm{~s}$, then washed with water for $30 \mathrm{~s}$, and dried with oil-free air spray (12).

Thereafter, 0.018-inch stainless steel lower incisor brackets (Gemini Roth System; 3M Unitek, Monrovia, CA, USA) were applied to the middle of the porcelain and composite disc surfaces using orthodontic primer and adhesive (Transbond XT; $3 \mathrm{M}$ Unitek). During bonding, to obtain a uniform adhesive thickness, each bracket was subjected to $300 \mathrm{~g}$ of force using a force gauge (Correx Co., Bern, Switzerland) for $10 \mathrm{~s}$ (13). A scaler was used to remove the excess resin overflow from the bracket base. Adhesives were polymerized using a LED light source (Elipar FreeLight 2; 3M ESPE) for a total of $40 \mathrm{~s}$, with $10 \mathrm{~s}$ for each of the mesial, distal, occlusal, and gingival surfaces.

\section{Table 1. Materials used in the present study}

\begin{tabular}{|lllll}
\hline Materials & Manufacturer & Material group code & Organic matrix & Fillers \\
\hline Vitadur Alpha & $\begin{array}{l}\text { Vita Zahnfabric, } \\
\text { Bad Säckingen, } \\
\text { Germany }\end{array}$ & Feldispatic ceramic & - & - \\
Filtek Ultimate & $\begin{array}{l}\text { 3M ESPE, St. Paul, } \\
\text { MN, USA }\end{array}$ & Nanofilled composite & $\begin{array}{l}\text { Bis-GMA, UDMA, } \\
\text { Bis-EMA, TEGDMA }\end{array}$ & $\begin{array}{l}20 \mathrm{~nm} \text { silica filler, 4-11 nm zirconia filler, } \\
\text { zirconia/silica cluster filler 78.5\% weight or } \\
63.3 \% \text { volume }\end{array}$ \\
Tetric EvoCeram & $\begin{array}{l}\text { Ivoclar Vivadent } \\
\text { AG, Schaan, } \\
\text { Liechtenstein }\end{array}$ & Nanohybrid composite & $\begin{array}{l}\text { Bis-GMA, UDMA, } \\
\text { Bis-EMA }\end{array}$ & $\begin{array}{l}\text { Barium glass, ytterbium-trifluoride, mixed } \\
\text { oxide, and copolymers } 75 \%-76 \% \text { weight } \\
\text { or } 53 \%-55 \% \text { volume }\end{array}$ \\
Gradia Direct Anterior & $\begin{array}{l}\text { GC Co., Tokyo, } \\
\text { Japan }\end{array}$ & Microhybrid composite & $\begin{array}{l}\text { UDMA, dimethacryate } \\
\text { comonomers }\end{array}$ & $\begin{array}{l}\text { Fumed silica, prepolymerized filler, silica } \\
\text { and/or fluoroaluminosilicate glass, 73\% weight }\end{array}$
\end{tabular}


The discs were embedded into $20 \mathrm{~mm} \times 10 \mathrm{~mm} \times 10 \mathrm{~mm}$ polymethyl methacrylate blocks (Meliodent; Heraeus Kulzer, Hanau, Germany), with the bracket bases placed parallel to the ground. All specimens were stored in $37 \pm 2{ }^{\circ} \mathrm{C}$ distilled water for $24 \mathrm{~h}$.

\section{Bracket Debonding Procedures}

The SBS was measured using a universal testing device (Autograph AGS-X, Shimadzu, Japan), where the value of the applied force is recorded to the electronic display of the device in Newtons $(\mathrm{N})$. The crosshead speed of the device was set to $1 \mathrm{~mm} / \mathrm{min}$ (14). The direction of the debonding force was applied to the ligature groove parallel to the bracket base (Figure 1). The force required to dislodge the bracket was recorded in N and converted to megapascals (MPa) using the following equation: Shear force $(\mathrm{MPa})=$ Debonding force $(\mathrm{N})$ / $(\mathrm{W} \times \mathrm{H})\left(\mathrm{mm}^{2}\right)$, where $\mathrm{W}$ is the width of the bracket base, and $\mathrm{H}$ is the height of the bracket base ( $1 \mathrm{Mpa}=1 \mathrm{~N} / \mathrm{m}^{2}$ ). A digital caliper was used to determine the bracket base area, which was found to be $10 \mathrm{~mm}^{2}$.
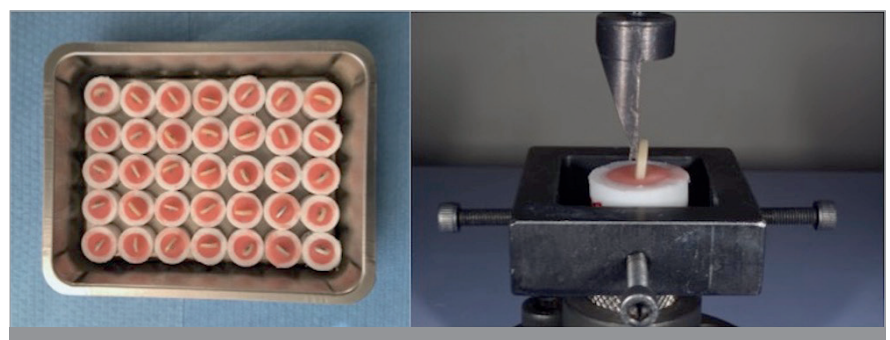

Figure 1. Shear bond testing of porcelain and composite discs embedded in polymethyl methacrylate blocks

\section{Determination of Fracture Sites}

After debonding, all disc surfaces were examined under a stereomicroscope at $10 \times$ magnification. The remaining residual adhesives on the disc surfaces were evaluated by the adhesive remnant index (ARI) defined by Artun and Bergland in 1984 (15). ARI scores range between 0 and 3 , with 0 indicating no adhesive remains on the disc surfaces; 1 indicating $<50 \%$ of adhesive remains on the disc surfaces; 2 indicating $>50 \%$ of adhesive remains on the disc surfaces; and 3 indicating that the entire adhesive remains on the disc surfaces.

\section{Statistical Analysis}

Descriptive statistics for studied variables (characteristics) were presented as mean, standard deviation, median, minimum, and maximum values. Normality test was performed, and violation of the normality assumption was detected. Kruskal-Wallis test was used to compare the aged and fresh groups with regard to SBS. Dunn's multiple comparison test was used to determine different groups. Chi-square or Fisher's exact tests were used to compare the aged and fresh groups with regard to ARI scores. The significance level was set to 5\%. Statistical Package for Social Sciences program version 13 (SPSS Inc.; Chicago, IL, USA) was used for all statistical computations.

\section{RESULTS}

Comparisons of SBSs in porcelain discs: The mean SBS values of metal orthodontic brackets in the aged and fresh VA porcelain

Table 2. Shear bond strength of metal orthodontic brackets bonded to aged and fresh porcelain and composite disc surfaces

\begin{tabular}{|c|c|c|c|c|c|c|}
\hline & & Sample size (n) & Median & Mean \pm SD & Minimum & Maximum \\
\hline \multirow[t]{2}{*}{ Vitadur Alpha } & Aging & 10 & 5.18 & $6.37 \pm 2.84 d^{*}$ & 4.00 & 12.05 \\
\hline & Fresh & 10 & 6.04 & $8.06 \pm 4.72 d$ & 3.67 & 16.79 \\
\hline Filtek Ultimate & Fresh & 10 & 8.89 & $10.85 \pm 3.11 c$ & 6.24 & 14.55 \\
\hline Tetric EvoCeram & Aging & 10 & 15.11 & $13.63 \pm 5.55 b c$ & 4.14 & 21.66 \\
\hline Gradia Direct Anterior & Fresh & 10 & 18.50 & $19.09 \pm 5.33 a$ & 10.38 & 26.92 \\
\hline Total & & 80 & 12.05 & $12.34 \pm 5.81$ & 3.67 & 26.92 \\
\hline
\end{tabular}

Table 3. Distribution of adhesive remnant index (ARI) scores among the aged and fresh groups

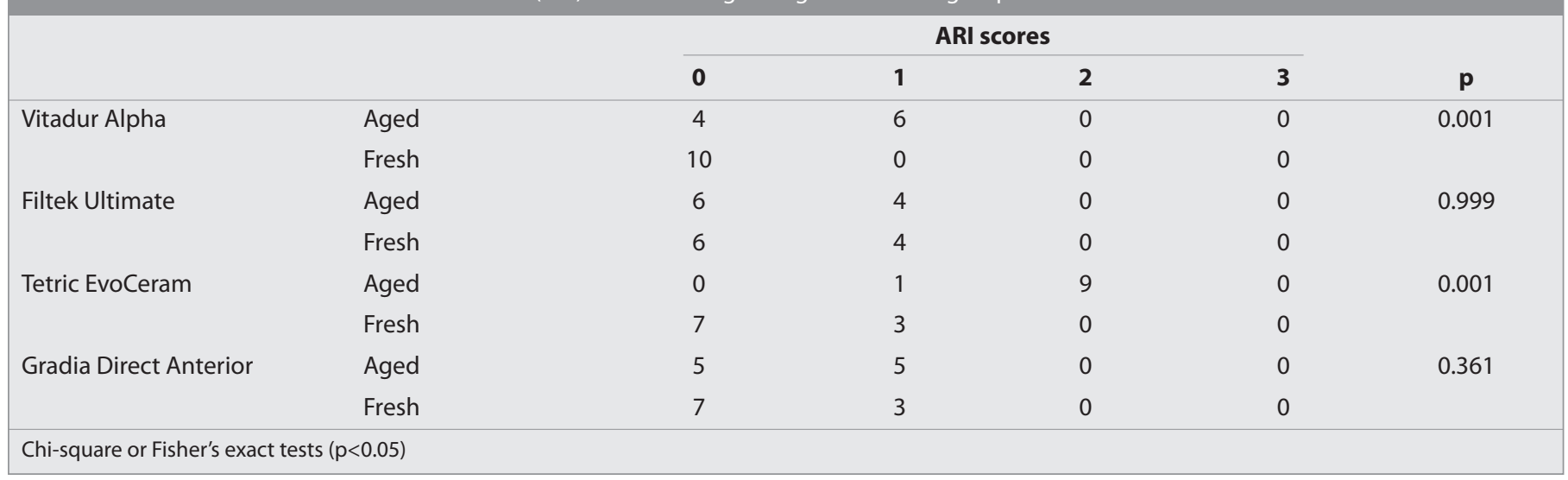


groups were $6.37 \pm 2.84$ and $8.06 \pm 4.72 \mathrm{MPa}$, respectively. The difference between the aged and fresh groups was not statistically significant (Table 2).

Comparisons of SBSs in composite discs: The mean SBS values of metal orthodontic brackets in the aged and fresh groups were 9.18 \pm 2.46 and 10.35 $\pm 3.11 \mathrm{MPa}$ for FU composite, 13.63 \pm 5.55 and $17.02 \pm 4.15 \mathrm{MPa}$ for TEC composite, and $15.01 \pm 3.67$ and $19.09 \pm 5.33$ MPa for GDA composite, respectively. Although the difference between the mean SBS of metal orthodontic brackets in the aged and fresh groups with FU and TEC composites was not statistically significant, the mean SBS of the fresh group with GDA composite was significantly higher. However, the differences between the mean SBS of metal orthodontic brackets with fresh GDA composite and fresh TEC composite and between the mean SBS of metal orthodontic brackets with aged GDA composite and aged and fresh TEC composites were not statistically significant (Table 2).

The results also show that the mean SBS of metal orthodontic brackets bonded to composite discs was higher than that of the porcelain discs.

ARI scores of porcelain discs: The ARI scores of VA porcelain were significantly higher in the aged group. Intergroup comparison showed that for VA porcelain, in the aged group, four samples had an ARI score of 0 , and six samples had an ARI score of 1, and all samples in the fresh group had 0 (Table 3 ).

ARI scores of composite discs: No significant difference was found between the ARI scores of the aged and fresh groups with FU and GDA composites, whereas the ARI score of the aged group with Tetric EvoCeram composite was higher. Intergroup comparison showed that for FU composite, six samples in the aged group had an ARI score of 0 , and four samples in the fresh group had an ARI score of 1. For Tetric EvoCeram composite, one sample had 1 and nine samples had 2 in the aged group, whereas seven samples had 0 and three samples had 1 in the fresh group. For GDA composite, five samples had 0 and five samples had 1 in the aged group, whereas seven samples had 0 and three samples had 1 in the fresh group (Table 3).

\section{DISCUSSION}

Dental restorations age in the long term due to the humid environment inside the mouth, since they become water-saturated and their free radical activity gradually dies off (1). The bond strengths of the aged materials decrease due to the hydrolytic degradation of the interface components and significant reduction in mechanical properties (16). In addition, the duration of aging and the material itself have a decisive effect on the decrease in bond strength $(10,11,17)$. In this context, the aim of the present study was to compare the SBS of metal orthodontic brackets bonded to aged and fresh porcelain (feldspathic ceramic) and composite (nanofilled, nanohybrid, and microhybrid) surfaces.

In the present study, storage in distilled water, a commonly used aging technique, was used as water behaves similar to the liquids that usually exist in the mouth and constantly interact with the teeth and restorations $(11,16,18)$. Furthermore, since the follow-up periods in prosthetic and restorative studies were between 3 and 22 years for composite restorations (19) and a minimum of 5 years for porcelain restorations (20), the duration of aging in our study was determined as the minimum follow-up period of 5 years.

To provide good adhesion and sustain the forces arising from mastication and orthodontic mechanotherapy, a minimum bond strength of 5-10 Mpa is recommended (21). In addition, extremely high bond strengths (40-50 MPa) should be avoided as they may harm the enamel due to the application of high debonding forces during or at the end of the treatment (22). It is assumed that the acceptable SBSs should be between $5 \mathrm{MPa}$ and $50 \mathrm{MPa}$, even if the limits are within theoretical parameters. The results of the present study reveal that the mean SBS values of metal orthodontic brackets in the aged and fresh groups were between $6.37 \pm 2.84$ and $19.09 \pm 5.33 \mathrm{MPa}$ for porcelain and composite discs, respectively, and these values were within the acceptable limits. The results also show that the SBS of metal orthodontic brackets bonded to composite discs was higher than those of porcelain discs.

Owing to their high translucency, feldspathic porcelains are relatively aesthetic and are frequently used $(23,24)$. A number of studies have been published to evaluate the SBS of metal orthodontic brackets bonded to fresh feldspathic porcelain discs (Vitadur Alpha; Vita Zahnfabrik, Bad Säckingen, Germany) that were subjected to different surface treatments using Transbond XT primer and adhesive (Transbond XT). Of these studies, Türk et al. (24) reported that the mean SBS of metal orthodontic brackets bonded to 12 feldspathic porcelain discs that underwent surface treatment with 9.6\% hydrofluoric acid (Porcelain Etch Gel; Pulpdent, Watertown, MA, USA) and silane agent (Bond Enhancer; Pulpdent) is $5.39 \pm 2.59 \mathrm{MPa}$. Yadav et al. (25) stated that the mean SBS of metal orthodontic brackets bonded to 20 feldspathic porcelain discs that underwent surface treatment with 9.5\% hydrofluoric acid (Ultradent) and silane agent (Kuraray Co., Osaka, Japan) is $9.9 \pm 2.7 \mathrm{MPa}$. Abdelnaby (26) determined that the mean SBS of metal orthodontic brackets bonded to 25 feldspathic porcelain discs that were subjected to surface treatment with 9.6\% hydrofluoric acid (Pulpdent) and resin-based primer (Embrace First-Coat) is $5.48 \pm 1.03 \mathrm{MPa}$. In addition, Buyuk et al. (5) indicated that the mean SBS of metal orthodontic brackets bonded to 10 feldspathic porcelain discs that were subjected to surface treatment with only $9.6 \%$ hydrofluoric acid (Ultradent) is $6.36 \pm 2.19 \mathrm{MPa}$. Consistent with these results, in our study, the mean SBS values of metal orthodontic brackets bonded to aged and fresh feldspathic porcelain discs were $6.37 \pm 2.84$ and 8.06 $\pm 4.72 \mathrm{MPa}$, respectively.

To our knowledge, no study in the literature has evaluated the SBS of metal orthodontic brackets bonded to aged feldspathic porcelain discs and compared the SBS of metal orthodontic brackets bonded to the aged and fresh groups. In the present study, no significant difference was found between the aged and fresh groups; therefore, the null hypothesis for porcelain surfaces was accepted. 
Nanofilled composites have been produced as universal restorative materials, and their strength and aesthetic properties allow the clinicians to use it for both anterior and posterior restorations $(17,27)$. A limited number of studies evaluated the SBS of metal orthodontic brackets bonded to aged and fresh nanofilled composite discs (Filtek ${ }^{\text {TM }}$ Supreme XT; 3M ESPE) on which Transbond XT primer and adhesive (Transbond XT) were applied. Among these, Bayram et al. (1) stated that the mean SBS of metal orthodontic brackets bonded to 15 nanofilled composite discs that were aged with an accelerated process involving surface treatment with $38 \%$ phosphoric acid (Pulpdent) is $3.71 \pm 1.22 \mathrm{MPa}$. Viwattanatipa et al. (27) reported that the mean SBS of metal orthodontic brackets bonded to 32 fresh composite discs that were subjected to surface treatment with 37\% phosphoric acid (3M ESPE Dental Products) and plastic conditioner agent (Reliance Orthodontic Product Inc.) is $4.03 \pm 1.6 \mathrm{MPa}$. In another study, it was found that the mean SBS of upper molar tubes bonded to 31 composite discs stored in deionized water for 1 month and were subjected to surface treatment with 9.6\% hydrofluoric acid, plastic conditioner agent (Reliance Orthodontic Product Inc.), and unite adhesive (3M Unitek) was $6.87 \pm 4.58 \mathrm{MPa}$ (28). To the best of our knowledge, there are no studies in the literature that have compared the SBS of metal orthodontic brackets bonded to aged and fresh nanofilled composite discs. In the present study, the mean SBS of metal orthodontic brackets bonded to aged and fresh nanofilled composite discs were $9.18 \pm 2.46$ and $10.35 \pm 3.11 \mathrm{MPa}$, respectively, and these values were higher than the results reported in the current studies on this subject. Furthermore, no significant difference was found between the aged and fresh groups.

Nanohybrid composites contain a range of macrofillers and microfillers in different sizes, which occupy the spaces between the larger particles and shorten the particle space (10). In studies evaluating the SBS of metal orthodontic brackets bonded to different nanohybrid composites, different results and different aging procedures were reported. Among these, Demirtas et al. (12) used thermocycling for aging and stated that the mean SBS of metal orthodontic brackets bonded to 40 nanohybrid composite discs (Filtek Z550; 3M ESPE) with a surface treatment of 37\% phosphoric acid (Pulpdent) and Transbond XT primer and adhesive (Transbond XT) is 4.58 $\pm 1.42 \mathrm{MPa}$. Hammad et al. (17) treated the labial surfaces of 20 incisor teeth with composite veneer restorations (Tetric EvoCeram; Ivoclar Vivadent AG, Schaan, Liechtenstein) and aged them in deionized water. Then, the mean SBS of metal orthodontic brackets bonded to these surfaces that were subjected to $38 \%$ phosphoric acid (3M ESPE) and no-mixed adhesive resin (Granitec ${ }^{\circledR}$; Confi-Dental Products Co., Louisville, CO, USA) surface treatments was calculated as 5.14 $\pm 0.03 \mathrm{MPa}$. Moreover, Eslamian et al. (29) calculated the mean SBS of metal orthodontic brackets bonded to labial surfaces of 15 premolar teeth restored with TEC composite and aged artificially and found that the mean SBS of metal orthodontic brackets is $12.85 \pm 5.20 \mathrm{MPa}$ when 5\% hydrofluoric acid (Ivoclar Vivadent AG) and no-mixed adhesive resin $\left(\right.$ Granitec $\left.^{\circledR}\right)$ were applied as surface treatments. To our knowledge, no study in the literature has evaluated the SBS of metal orthodontic brackets bonded to fresh nanohybrid composite discs and compared the aged and fresh groups. In our study, the mean SBS values of metal orthodontic brackets were
13.63 \pm 5.55 and $17.02 \pm 4.15 \mathrm{MPa}$ in the aged and fresh groups, respectively, with no significant difference between the groups. Therefore, the null hypothesis for composite surfaces was rejected for nanofilled FU and nanohybrid TEC composites.

In our study, it was also observed that the mean SBS values of metal orthodontic brackets bonded to aged and fresh microhybrid GDA composite discs were $15.01 \pm 3.67$ and 19.09 \pm 5.33 , respectively, and the mean SBS of the fresh group was significantly higher. Therefore, the null hypothesis for composite surfaces was accepted for microhybrid GDA composite. Although a number of studies have been published to evaluate the SBS of metal orthodontic brackets bonded to different aged and fresh microhybrid composites with Transbond XT primer and adhesive surface treatments, to our knowledge, no study in the literature has evaluated the microhybrid GDA composite. Tayebi et al. (4) evaluated the cyclic aged microhybrid Filtek Z250 composite discs (3M ESPE) and concluded that the mean SBS values of metal orthodontic brackets bonded to these composite discs are $9.94 \pm 2.5$ and $7.57 \pm 4.0$ Mpa in the groups that underwent sandblasting and burring as surface treatments, respectively. In his study, Tse (6) used Filtek Z250 microhybrid composite in the restoration of Class V buccal cavities of 24 upper incisor teeth and aged these restorations in distilled water. Then, the mean SBS of metal orthodontic brackets bonded to these aged composites was calculated and found as 12.1 $\pm 3.4 \mathrm{MPa}$. Moreover, Brunharo et al. (30) stated that the mean SBS of metal orthodontic brackets bonded to 10 fresh Charisma microhybrid composite discs (Heraeus Kulzer) that were subjected to $37 \%$ phosphoric acid surface treatment is $5.82 \pm 1.90 \mathrm{MPa}$.

In the present study, the ARI scores showed that bond failure predominantly occurred between the restoration and the adhesive as the majority of the adhesive remained on the bracket bases in all groups except for aged Tetric EvoCeram composite. These results were consistent with previous studies $(1,6,24,26)$. However, in the aged TEC composite, most of the adhesive remained on the restoration surface, which was consistent with the results by Demirtas et al. (12) evaluating the aged nanohybrid composite discs (Filtek Z550; 3M ESPE).

Finally, we compared the SBS of metal orthodontic brackets in one porcelain material and three different composite materials that were subjected to a single surface treatment. Therefore, further studies using a greater number of porcelain and composite materials with different surface treatments are recommended.

\section{CONCLUSION}

- No significant difference was found between the aged and fresh groups with VA porcelain and FU and TEC composites with regard to the SBS of metal orthodontic brackets.

- The SBS of metal orthodontic brackets was significantly higher in the fresh group with GDA composite.

- No significant difference was found between the aged and fresh groups with FU and GDA composites with regard to ARI scores.

- $\quad$ ARI scores were significantly higher in the aged group with VA porcelain and TEC composite. 


\section{Ethics Committee Approval: N/A.}

\section{Informed Consent: N/A.}

Peer-review: Externally peer-reviewed.

Author Contributions: Concept - Y.K., B.Ü.D.; Design - Y.K., B.Ü.D.; Data Collection and/or Processing - Y.K., B.Ü.D., A.D.; Analysis and/or Interpretation - Y.K.; Literature Search - Y.K.; Writing Manuscript - Y.K.; Critical Review - Y.K.

Conflict of Interest: The authors have no conflict of interest to declare.

Financial Disclosure: The authors declared that this study has received no financial support.

\section{REFERENCES}

1. Bayram M, Yesilyurt C, Kusgöz A, Ulker M, Nur M. Shear bond strength of orthodontic brackets to aged resin composite surfaces: effect of surface conditioning. Eur J Orthod 2011; 33: 174-9. [CrossRef]

2. Scribante A, Sfondrini MF, Fraticelli D, Daina P, Tamagnone A, Gandini P. The influence of no-primer adhesives and anchor pylons bracket bases on shear bond strength of orthodontic brackets. BioMed Res Int 2013; DOI: 10.1155/2013/315023. [CrossRef]

3. Al Jabbari YS, Al Taweel SM, Al Rifaiy M, Algahtani MQ, Koutsoukis $T$, Zinelis $S$. Effects of surface treatment and artificial aging on the shear bond strength of orthodontic brackets bonded to four different provisional restorations. Angle Orthod 2014; 84: 649-55. [CrossRef]

4. Tayebi A, Fallahzadeh F, Morsaghian M. Shear bond strength of orthodontic metal brackets to aged composite using three primers. J Clin Exp Dent 2017; 9: 749-55. [CrossRef]

5. Buyuk SK, Kucukekenci AS. Effects of different etching methods and bonding procedures on shear bond strength of orthodontic metal brackets applied to different CAD/CAM ceramic materials. Angle Orthod 2018; 88: 221-6. [CrossRef]

6. Tse M. The effect of surface treatments and bonding agents on the shear bond strength of orthodontic brackets bonded to aged composite restorations. Thesis 2012.

7. Ozcan M, Barbosa SH, Melo RM, Galhano GA, Bottino MA. Effect of surface conditioning methods on the microtensile bond strength of resin composite to composite after aging conditions. Dent Mater 2007; 23: 1276-82. [CrossRef]

8. Rocha RS, Oliveira AC, Canneppele TMF, Bresciani E. Effect of artificial aging protocols on surface gloss of resin composites. Int J Dent 2017; DOI:1155/2017/3483171.

9. Liebermann A, Ilie N, Roos M, Stawarczyk B. Effect of storage medium and aging duration on mechanical properties of self-adhesive resin-based cements. J Appl Biomater Funct Mater 2017; 15: 206-14. [CrossRef]

10. Schmidt C, llie N. The effect of aging on the mechanical properties of nanohybrid composites based on new monomer formulations. Clin Oral Investig 2013; 17: 251-7. [CrossRef]

11. Alshali RZ, Salim NA, Satterthwaite JD, Silikas N. Long-term sorption and solubility of bulk-fill and conventional resin composites in water and artificial saliva. J Dent 2015; 43: 1511-8. [CrossRef]

12. Demirtas HK, Akin M, lleri Z, Basciftci FA. Shear-bond-strength of orthodontic brackets to aged nano-hybrid composites-resin surfaces using different surface preparation. Dent Mater J 2015; 34: 86-90. [CrossRef]
13. Bishara SE, Ajlouni R, Laffoon JF, Warren JJ. Comparison of shear bond strength of two self-etch primer/adhesive systems. Angle Orthod 2006; 76: 123-6.

14. Cehreli SB, Guzey A, Arhun N, Cetinsahin A, Unver B. The effects of prophylactic ozone pretreatment of enamel on shear bond strength of orthodontic brackets bonded with total or self-etch adhesive systems. Eur J Dent 2010; 4: 367-73.

15. Artun J, Bergland S. Clinical trials with crystal growth conditioning as an alternative to acid-etch enamel pretreatment. Am J Orthod 1984; 85: 333-40. [CrossRef]

16. Costa AR, Correr AB, Consani S, Giorgi MC, Vedovello SA, Vedovello Filho $M$, Santos EC, Correr-Sobrinho L. Influence of water storage and bonding material on bond strength of metallic brackets to ceramic. Braz Dent J 2015; 26: 503-6. [CrossRef]

17. Hammad SM, El Banna MS. Effects of cyclic loading on the shear bond strength of metal orthodontic brackets bonded to resin composite veneer surface using different conditioning protocols. Prog Orthod 2013; 23: 14. [CrossRef]

18. Yuasa T, lijima M, Ito S, Muguruma T, Saito T, Mizoguchi I. Effects of long-term water storage and thermocycling on bond strength of two self-etching primer adhesive system. Eur J Orthod 2010; 32: 285-90. [CrossRef]

19. Borgia E, Baron R, Borgia JL. Quality and survival of direct light-activated composite resin restorations in posterior teeth: A 5- to 20-year retrospective longitudinal study. J Prosthodont 2017; DOI: 10.1111/ jopr.12630. [CrossRef]

20. Araujo NS, Moda MD, Silva EA, Zavanelli AC, Mazaro JV, Pellizzer EP. Survival of all-ceramic restorations after a minimum follow-up of five years: A systematic review. Quintessence Int 2016; 47: 395-405.

21. Scribante A, Contreras-Bulnes R, Montasser MA, Vallittu PK. Orthodontics: Bracket materials, adhesives systems, and their bond strength. BioMed Res Int 2016; 2016: 1329814. [CrossRef]

22. Giannini M, Soares CJ, de Carvalho RM. Ultimate tensile strength of tooth structures. Dent Mater 2004; 20: 322-9. [CrossRef]

23. Mehta AS, Evans CA, Viana G, Bedran-Russo A, Galang-Boquiren MT. Bonding of metal orthodontic attachments to sandblasted porcelain and zirconia surfaces. BioMed Res Int 2016; 5762785. [CrossRef]

24. Türk T, Saraç D, Saraç YS, Elekdağ-Türk S. Effects of surface conditioning on bond strength of metal brackets to all-ceramic surfaces. Eur J Orthod 2006; 28: 450-6. [CrossRef]

25. Yadav S, Upadhyay M, Borges GA, Roberts WE. Influence of ceramic (feldspathic) surface treatments on the micro-shear bond strength of composite resin. Angle Orthod 2010; 80: 765-70. [CrossRef]

26. Abdelnaby YL. Effects of cyclic loading on the bond strength of metal orthodontic brackets bonded to a porcelain surface using different conditioning protocols. Angle Orthod 2011; 81: 1064-9. [CrossRef]

27. Viwattanatipa N, Jermwiwatkul W, Chintavalakorn R, Nanthavanich $\mathrm{N}$. The effect of different surface preparation techniques on the survival probabilities of orthodontic brackets bonded to nanofilled composite resin. J Orthod 2010; 37: 162-73. [CrossRef]

28. Viwattanatipa N, Prasertsangwal J, Juntavee N. Weibull analysis of shear/peel bond strength of orthodontic buccal tubes bonded to five resin composites. Orthod Waves 2008; 67: 120-7. [CrossRef]

29. Eslamian L, Borzabadi-Farahani A, Mousavi N, Ghasemi A. The effects of various surface treatments on the shear bond strengths of stainless steel brackets to artificially-aged composite restorations. Aust Orthod J 2011; 27: 28-32.

30. Brunharo IH, Fernandes DJ, De Miranda MS, Artese F. Influence of surface treatment on shear bond strength of orthodontic brackets. Dental Press J Orthod 2013; 18: 54-62. [CrossRef] 